\title{
ESOC8-0318 AS20-016 \\ ICHO score: a new score to predict functional outcome in patients with intracerebral hemorrhage
}

Y. Silva, M. Terceño, J. Puig, S. Bashir, I Bragado, P. Daunis-i-Estadella, S. Pedraza, J. Serena Stroke Unit. Department of Neurology. Hospital Dr Josep Trueta, Girona Biomedical Research Institute (IDIBGI), University of Girona, Department of computer science, Girona

Background and Aims: Intracerebral hemorrhage is a leading cause of strokerelated morbidity and mortality. We propose a model based on clinical and imaging parameters to predict functional outcome: the ICHO score.

Methods:

\begin{tabular}{|l|l|}
\hline Inclusion criteria & Secondary ICH \\
Primary ICH & mRs $>2$ \\
$<12$ hours & Infratentorial ICH \\
Informed consent & Terminal illness or coma \\
\cline { 2 - 3 } &
\end{tabular}

Clinical variables

Blood pressure and glucose NiHSS at admission, $24 \mathrm{~h}, 72 \mathrm{~h}, 3 \mathrm{~m}$ Functional outcome: mRs 3 months

Radiological variables
Location ICH
Volumes of hematoma admission, $24 \mathrm{~h}, 72 \mathrm{~h}$
Volumes of perihematomal edema admission,
$24 \mathrm{~h}$ and 72 hours
(Olea Sphere 3.0, La Ciotat, France)
Intraventricular extension

Independent predictors of poor outcome were identified by multiple regression analysis. The best model was selected by Bayesian information criterion.

\begin{tabular}{|llc|}
\hline & $m R s>2$ & $m R s \leq 2$ \\
Age & $n=184$ & $n=96$ \\
NIHSS ad & $17(12-19)$ & $5(3-9)$ \\
BP & $148(134-164)$ & $143(128-152)$ \\
Glucose & $114(101-139)$ & $105(95-116)$ \\
Vol ICH & $24(10-43)$ & $7(2,5-12)$ \\
Vol ICH 72 & h29 (12-56) & $7(3,4-13)$ \\
PHE Vol & $11(5-25)$ & $4,6(1,3-9)$ \\
PHE Vol $7230(14,5-53)$ & $10(5-17)$ \\
\hline
\end{tabular}

Results: 280 patients

The best predictors were Age

NIHSS $24 \mathrm{~h}$

Hematoma volume $72 \mathrm{~h}$

ICHO score $=-0,837+(0,032 *$ age $)+$ $(0,152 *$ NIHSS24h)+ $0,011^{*}$ hematoma volume $72 \mathrm{~h}$ )

Conclusions: The ICHO score might be a valid clinical grading scale for 3-months functional outcome after $\mathrm{ICH}$. Further validation in different populations is needed 